\title{
Validation of a Wireless Portable Biofeedback System for Balance Control: Preliminary Results
}

\author{
Laura Rocchi, Marco Benocci, Elisabetta Farella, Luca Benini, Lorenzo Chiari, \\ Department of Electronics, Computer Science and Systems \\ University of Bologna \\ Viale Risorgimento 2, 40136 Bologna, Italy \\ [1.rocchi/marco.benocci/elisabetta.farella /luca.benini/lorenzo.chiari]@unibo.it
}

\begin{abstract}
The present study describes the results regarding the preliminary validation of a system for rehabilitation of balance control that integrates the most recent technology advances with the latest findings about motor control and rehabilitation-engineering. The biofeedback system is based on a palmtop computer and a body sensor network, with a modular architecture, both hardware and software. Validation was performed on young healthy subjects, simulating impairment to the balance control system at the proprioception level. Promising results were obtained by means of the biofeedback system, both in terms of adequacy and usability of the system, and in terms of improvement of the balance performance.
\end{abstract}

\section{INTRODUCTION}

In recent years, principles of pervasive solutions and tailored interventions for healthcare are gaining significant relevance, both in the scientific and in the care-providers and clinical community, with a potential, significant impact on the society and the healthcare systems. This new approach has been primarily possible thanks to the recent technology advances in several disciplines, such as telecommunications, electronics, computer science and real-time data analysis. In particular, wireless communications, miniaturized sensors, and low power design have opened new perspectives for relevant biomedical applications, especially when unobtrusiveness and ubiquitous availability are critical requirements. One field that can significantly take advantage from such approach is the field of motion tracking for movement and balance assessment and rehabilitation. Motor and balance impairments are crucial symptoms in neurodegenerative diseases (such as Parkinson's Disease [1]), in musculoskeletal damages, and in the elderly [2], with a massive impact on the quality of life of the patients. Motor and balance optimization is also crucial to maintain a healthy lifestyle (in the perspective of preventive medicine and public health) [3], and in the view of performance enhancement in sports [4]. In addition, in several diseases, motor and balance rehabilitation and improvement can help preventing severe and disabling side effects [5].

Inertial sensors (accelerometers in particular) have proven to be essential elements in applications for movement and

Permission to make digital or hard copies of all or part of this work for personal or classroom use is granted without fee provided that copies are not made or distributed for profit or commercial advantage and that copies bear this notice and the full citation on the first page. To copy otherwise, to republish, to post on servers or to redistribute to lists, requires prior specific permission and/or a fee.

PERVASIVEHEALTH 2008, 30 Jan - 1 Feb. Tampere, Finland

Copyright $\odot$ 2008 ICST 978-963-9799-15-8

DOI 10.4108/ICST.PERVASIVEHEALTH2008.2532 balance control, because of their small size, portability and the useful kinematic information they supply [6].

In this scenario, recently a system for rehabilitation and improvement of balance and posture has been proposed. Such system is based on motion tracking, real-time data processing and biofeedback restitution to the user, through an audio signal (audio-biofeedback, ABF) [7]. In previous studies [8], the proposed biofeedback system was proven to be effective for balance improvement in patients with bilateral vestibular loss.

In the present study, an advancement of the former system is described and validated on a healthy population. Such biofeedback system may, in perspective, respond to the lack, on the market and in the clinical and research practice, of biofeedback systems which are wearable, easily accessible by users, unobtrusive, and cost effective. This new version avoids any wired link among system components, resulting in a wireless body area network that integrates both sensors and actuators for long-term monitoring and biofeedback. The integration of a palmtop computer as a wearable, wireless, general-purpose node enables the implementation of dedicated applications for biofeedback. The audio biofeedback chosen is provided through a lightweight headset. The modular system architecture allows large flexibility in the variety and number of sensors that can be included, to enable future developments in many different biomedical applications. Sensing nodes characterized by totally wireless communications are essential also for the development of applications for motion and balance tracking and rehabilitation that are underway, since such applications imply large user's movements and distal sensors positioning, making wireless connections a fundamental feature of the system.

\section{WIRELESS BIOFEEDBACK SYSTEM DESIGN}

The portable and wireless biofeedback system was designed to include: i) modular hardware architecture for diverse sensors integration and wireless communications; ii) data acquisition architecture to manage a wireless body area network of sensor nodes; iii) modular software design to allow easy and optimized integration of different biofeedback algorithms and feedback restitution. 


\section{A. Hardware Architecture}

The biofeedback system is based, so far, on a unique sensor positioned on the trunk to detect posture and monitor subject's sway on the horizontal plane. The biofeedback restitution is obtained by means of a stereo sound provided by headsets. However, the system was design to potentially include a network of several and different sensing nodes, to provide different signals for biofeedback restitution (e.g. tactile, visual).

In its present release, the wireless body area network consists of three kinds of nodes: (i) a sensing node for data acquisition, (ii) an actuator node to provide the ABF signal, (iii) a processing node, namely a palmtop computer, in this version of the system) which acts as a general purpose node, with intelligence on-board and storage capability. The processing node manages the data from the body sensor, in order to estimate the user's posture and motion, and to provide adequate audio-feedback.

The sensing node (Fig. 1) adopts a modular architecture. It is organized in layers, corresponding to functional blocks to optimize node size and to facilitate upgrade of the components. The main layer is equipped with a microcontroller (ATMEL ATmega8), which provides a good trade-off between performance (1 MIPS / MHz) and low power consumption, and with the sensor: a MEMS tri-axial, digital output, linear accelerometer (STM LIS3LV02DQ). The accelerometer range is $\pm 2 \mathrm{~g}$ and it is able to measure accelerations over a bandwidth of $640 \mathrm{~Hz}$ for each axis.

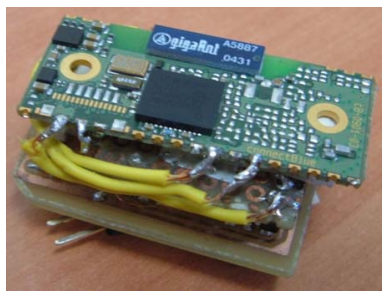

Fig. 1. Sensing node

The wireless communications is provided by a Bluetooth module (ConnectBlue, CB-OEMSPA311i-04) to enable communication with the palmtop computer. The system is a Bluetooth ${ }^{\circledR}$ 2.0, with SPP interface implemented into the firmware, capable of high Data Rate up to $3 \mathrm{Mbps}$. The power consumption is $0.9 \mathrm{~mW}$ in sleep mode and it requires a maximum of $150 \mathrm{~mW}$ in transmission mode. The power supply layer consists in a Li-Ion battery at $4.2 \mathrm{~V}$ with a stabilization circuit to improve power consumption. Total node power consumption is about $180 \mathrm{~mW}$.

The considered processing node is the HP iPAQ $h \times 4700$ with Bluetooth capabilities operating with Windows CE 4.2 OS; Intel ${ }^{\circledR}$ PXA270 processor $(624 \mathrm{MHz})$, suitable for realtime signal processing. The palmtop computer allows to perform data logging and to store data in text format on an SD cards; therefore, the data have fast and simple portability to an external device for offline signal analysis. The audio-feedback is provided by a stereo headset Sennheiser HD201 with a 21$18 \mathrm{KHz}$ Frequency response $(-3 \mathrm{~dB}$ rel. $1 \mathrm{KHz})$.
Accelerometer data are sampled at $20 \mathrm{~Hz}$ (sampling frequency may potentially increase up to $100 \mathrm{~Hz}$ ); the output stereo sound is updated every $50 \mathrm{~ms}$, optimized according to the physiological users' responses $[2,3]$.

\section{B. Software Architecture}

The software architecture has been designed to best accomplish: i) real-time data processing; ii) input-output synchronization; iii) trouble-free integration of further biofeedback algorithms; (iv) independence from sensor node(s) characteristics and set-up. Such features have been combined with a user-friendly graphical interface for non expert users. The interface offers the possibility to select the appropriate biofeedback algorithm and user-specific options, and to perform a spot-check on the sensing node correct functioning and position. Furthermore, the software allows the detection of possible radio communication problems, notifying the user in case of misuse of the system.

\section{AUDIO BIOFEEDBACK ALGORITHM}

The sensing node measures trunk planar accelerations (in the anteroposterior, AP, and mediolateral, ML, directions), including the gravitational component, as an estimate of the sway of the user's body centre of mass. Such information is than processed by the audio-feedback generation algorithm that maps the movements and acceleration of the centre of mass to an audio signal. As a consequence, all the information available regarding relative position and movement direction of the user's trunk are coded into a stereo sound in terms of frequency modulation, volume variation, and L/R balance of the stereo sound provided by the headset.

The frequency codes the value of the instantaneous acceleration in the AP direction, through a piecewise-linear function, represented in Fig. 2A. The volume increases, with a sigmoid law, with the distance from the Target Region (TR, Fig. 2A). The TR is defined by a range of acceleration values, subject-specific and identified during the first 10 seconds of each trial (calibration phase), which are considered physiological for the user, since they reflect the small ballisticlike movements, typical of the postural control system [9]. In the TR the volume and frequency of the ABF sound are constant, at particularly comfortable levels [7]. The user's task, while using the ABF system, is to maintain the trunk acceleration (that approximates the position) inside the TR. The dynamics of the sound generation is determined by the TR and by the Limit Region (LR). Previous results [7;8] and experimental sessions allowed us to set TR and LR respectively as 1.50 and 10 times the standard deviation of the acceleration in the calibration phase. The user's acceleration in the ML direction is coded by left/right volume balance modulation of the stereo sound, through an exponential function (Fig. 2B).

Every $50 \mathrm{~ms}$, the biofeedback sound is updated according to the specific functions represented in Fig. 2. For further details on the ABF algorithm and functions, refer to. [7]. 

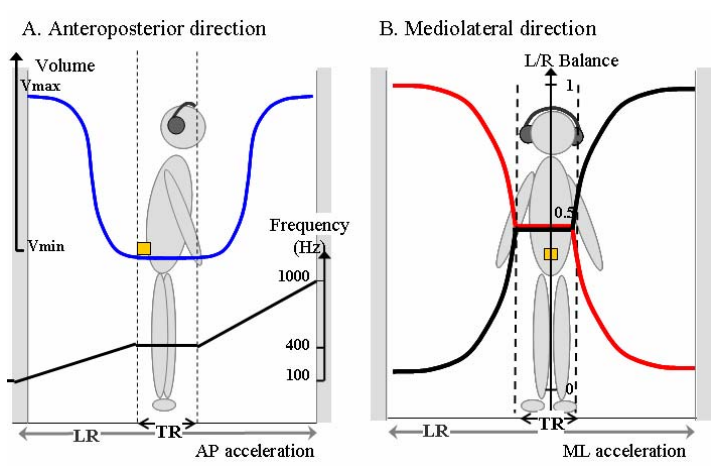

Fig. 2. Audio biofeedback sound dynamics. A. Frequency and volume modulation based on AP acceleration. B. Balance of the stereo sound based on the ML acceleration

\section{Audio BiofEedback System VAlidation}

\section{A. Experimental session: Methods}

Experimental set-up. The validation of the system was performed by means of experimental sessions that involved healthy young subjects, which performed trials of balance both with and without the use of the ABF system. In this first phase of the validation process, since healthy subjects were involved, impairment to the sensory and proprioceptive system of the lower leg was simulated by means of alterations to the afferent sensory system using an unstable support, i.e. a foam-rubber ( $9 \mathrm{~cm}$ thick) beneath the feet of subjects. .

Eight subjects were considered (age 21-36 yrs; height: 1.60-1.80 m; weight: $57-75 \mathrm{Kg}$ ), with no history of muscleskeletal disorders. Subjects were asked to maintain a standing normal postural position, for 60 seconds with eyes closed, arms crossed on chest and feet in a natural position (Fig. 3). Each subject performed 3 different kinds of trials: 1) Baseline: normally standing on a firm surface; 2) Sensory perturbation: standing on the unstable support; 3) Sensory perturbation $+A B F$ : standing on the unstable support, with the aid of the ABF system. Each condition was repeated 4 times, and trials administered randomly, to minimize the influence of learning or fatigue effects. At the beginning of each trial during the 10s calibration phase, the TR was calculated, and then used in real-time in case trials with the ABF system.

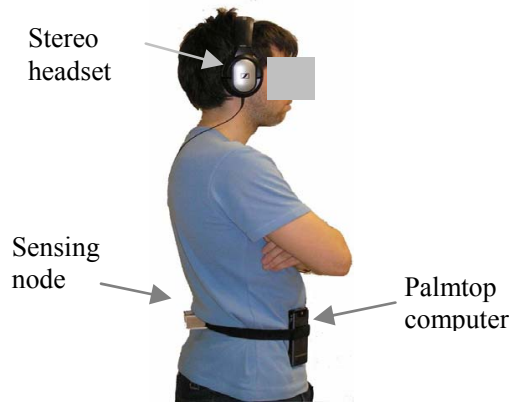

Fig. 3. Experimental set-up

Data analysis. Data were acquired and considered in postprocessing to evaluate the behavior of subjects during the different testing conditions, and to quantify the possible effect of the ABF system. Data from the accelerometer were considered, along the ML and AP direction. A low-pass filter was applied (zero-phase, cut-off frequency of $1 \mathrm{~Hz}$ ), to approximately estimate the inclination of the trunk, to quantify the oscillation of the body (in particular of the center of mass) during quiet standing (postural sway), that describes the level of postural stability [1;9]. The baseline condition was considered to evaluate possible anomalies in subjects' behavior and to obtain confirmation of the destabilizing effect of the unstable support. Several parameters to quantify postural sway were considered. The most informative parameters for this particular application (see [8]), then retained for further analyses, were: the percentage of time spent inside the TR (\%TIME_TR); the standard deviation $(S T D)$ of the filtered accelerometric signals, that quantifies the amount of the postural sway. The parameters were computed both for the AP and ML direction.

\section{B. Experimental session: Results}

The device was judged easy-to-use and comfortable by the subjects involved in the validation sessions, who learned to use it properly after only few minutes of training.

Comparison between Baseline and Sensory-perturbation conditions (without biofeedback) confirmed that the unstable base of support perturbed the balance of subjects and it was then considered a good simulation of proprioception and sensory impairments (see Fig. 4A-B).

With the unstable base of support, on the average, the use of the ABF system allowed the maintenance of a better level of balance (see Fig. 4B-C). In Fig. 4 an example of the behavior of a representative subject in the three testing conditions is shown $(1 \mathrm{~Hz}$ filtered and not filtered accelerometric data, in AP direction).

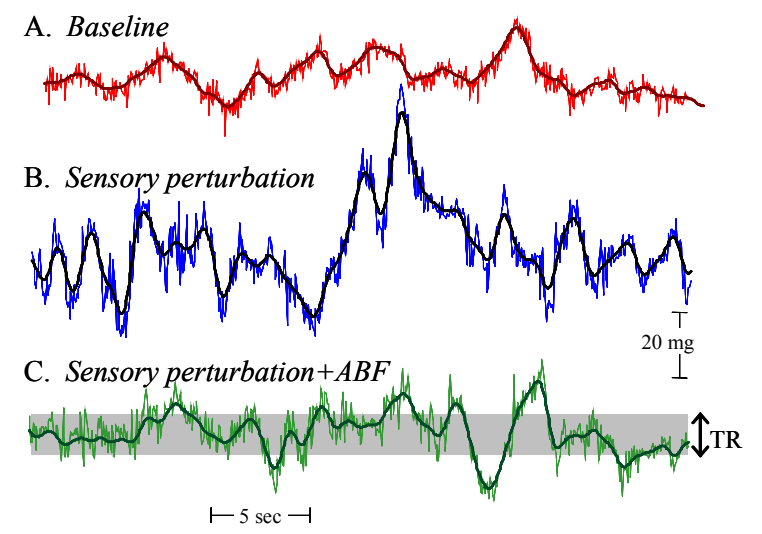

Fig. 4. Anteroposterior acceleration (not filtered and $1 \mathrm{~Hz}$-filtered) for a representative subject. A. Baseline (firm surface). B. Sensory perturbation (unstable base of support). C. Sensory perturbation and $\mathrm{ABF}$, with the correspondent TR.

A meticulous single-subject analysis was performed, highlighting the following considerations: (i) one of the 8 subjects was not actually able to adequately interpret the audio signal coming from the $\mathrm{ABF}$ system, and was then 
excluded from further analyses; (ii) 6 subjects showed improvement in balance performances in AP direction; (iii) 3 subjects showed improvement in the ML direction, (confirming a better ABF efficacy in the AP than in the ML direction); (iv) one subject did not change the \%TIME TR from the condition without to the condition with $\overline{A B F}$, however he/she showed a general reduction of STD, highlighting a non-specific but present reduction of the postural sway.

The parameters retained for analyses (\%TIME_TR and $S T D$ ) are shown in Fig. 5 for each subject (after a within subject average for each condition). The previous singlesubject considerations may be deducted also from such figure. It is clear that, comparing the two Sensory-perturbation conditions, \%TIME_TR consistently increased when the ABF was used compared to condition without it, and that, simultaneously, the STD tended to decrease. These results revealed a major direction-specific control of the postural sway and a general decreasing trend of the postural sway, when using $\mathrm{ABF}$ in sensory impaired conditions. Further statistical analysis will be performed (grouping the subjects) when a larger population will be available.
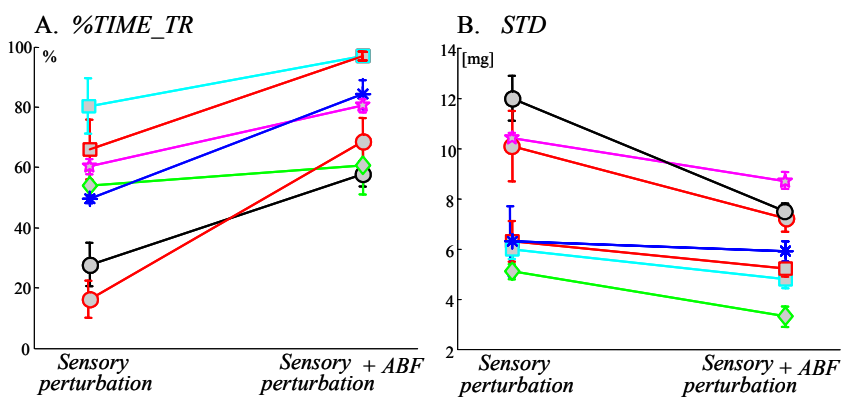

Fig. 5 Parameters quantifying postural control in sensory perturbed condition, with and without the $\mathrm{ABF}$

\section{CONCLUSION}

The proposed wireless system may represent an ubiquitous tool for rehabilitation of balance control. The modular architecture enables its use in several different contexts, with different kind of users (e.g. healthy elderly for physical activity maintenance, frail elderly for fall prevention, Parkinson's patients for posture correction), in different environments (e.g. at home, outdoor, in the clinic), with different feedback restitutions (e.g. audio, video, haptic), through the integration of different algorithms and sensor nodes in a wireless body area network. Results from the validation process are promising, though they suggest a possible misunderstanding, in some subjects, of information coming from the stereo balance sound modulation. However, further experiments are necessary, both on a healthy population and on populations with impairments to the motor control system.

Upcoming developments will include information from the sensing node on the trunk integrated with data collected from additional body sensors and related to further aspects of posture and movement. In addition, different biofeedback algorithms, customized for different population and motor tasks, will be implemented and tested.

\section{REFERENCES}

[1] Rocchi, L., Chiari, L., and Horak, F. B., "Effects of deep brain stimulation and levodopa on postural sway in Parkinson's disease," J. Neurol. Neurosurg. Psychiatry, vol. 73, pp. 267-274, Sept.2002.

[2] Schultz, A. B., "Mobility impairment in the elderly: challenges for biomechanics research," J. Biomech., vol. 25, pp. 519-528, May1992.

[3] Maki, B. E., Holliday, P. J., and Topper, A. K., "A prospective study of postural balance and risk of falling in an ambulatory and independent elderly population," J. Gerontol., vol. 49, pp. M72-M84, Mar.1994.

[4] Carrick FR Professor Of Neurology, Oggero, E., Pagnacco, G., Brock, J. B., and Arikan, T., "Posturographic testing and motor learning predictability in gymnasts," Disabil. Rehabil., pp. 1-9, Feb.2007.

[5] Protas, E. J., Mitchell, K., Williams, A., Qureshy, H., Caroline, K., and Lai, E. C., "Gait and step training to reduce falls in Parkinson's disease," NeuroRehabilitation., vol. 20, pp. 183-190, 2005.

[6] Mathie, M. J., Coster, A. C., Lovell, N. H., and Celler, B. G., "Accelerometry: providing an integrated, practical method for long-term, ambulatory monitoring of human movement," Physiol. Meas., vol. 25, pp. R1-20, Apr.2004.

[7] Chiari, L., Dozza, M., Cappello, A., Horak, F. B., Macellari, V., and Giansanti, D., "Audio-Biofeedback for Balance Improvement: An Accelerometry-Based System," Biomedical Engineering, IEEE Transactions on, vol. 52, pp. 2108-2111, 2005.

[8] Dozza, M., Chiari, L., and Horak, F. B., "Audio-biofeedback improves balance in patients with bilateral vestibular loss," Arch. Phys. Med. Rehabil., vol. 86, pp. 1401-1403, July2005.

[9] Winter, D. A., Prince, F., Frank, J. S., Powell, C., and Zabjek, K. F., "Unified theory regarding $\mathrm{A} / \mathrm{P}$ and $\mathrm{M} / \mathrm{L}$ balance in quiet stance," $\mathrm{J}$. Neurophysiol., vol. 75, no. 6, pp. 2334-2343, June1996. 